\title{
Existence and Uniqueness Theorem of Fractional Mixed Volterra-Fredholm Integrodifferential Equation with Integral Boundary Conditions
}

\author{
Shayma Adil Murad, ${ }^{1}$ Hussein Jebrail Zekri, ${ }^{2}$ and Samir Hadid ${ }^{3}$ \\ ${ }^{1}$ Department of Mathematics, Faculty of Science, Dohuk University, Kurdistan, Iraq \\ 2 Department of Mathematics, Faculty of Science, Zakho University, Kurdistan, Iraq \\ ${ }^{3}$ Department of Mathematics and Science, College of Education and Basic Sciences, Ajman University of \\ Science and Technology, UAE
}

Correspondence should be addressed to Samir Hadid, samir_hadid@yahoo.com

Received 7 May 2011; Accepted 24 May 2011

Academic Editor: Shaher Momani

Copyright (c) 2011 Shayma Adil Murad et al. This is an open access article distributed under the Creative Commons Attribution License, which permits unrestricted use, distribution, and reproduction in any medium, provided the original work is properly cited.

We study the existence and uniqueness of the solutions of mixed Volterra-Fredholm type integral equations with integral boundary condition in Banach space. Our analysis is based on an application of the Krasnosel'skii fixed-point theorem.

\section{Introduction}

In the last century, notable contributions have been made to both the theory and applications of the fractional differential equations. For the theory part, Momani and Hadid have investigated the local and global existence theorem of both fractional differential equation and fractional integrodifferential equations; see [1-6]. Fractional-order differential equations have recently proved to be valuable tools in the modeling of many phenomena in various fields of science and engineering.

Integrodifferential equations with integral boundary conditions are often encountered in various applications; it is worthwhile mentioning the applications of those conditions in the study of population dynamics and cellular systems. For a detailed description of the integral boundary conditions, we refer the reader to a recent paper [7]. In [8], Tidke studied the problem of existence of global solutions to nonlinear mixed Volttera-Fredholm integrodifferential equations with nonlocal condition.

Ahmad and Nieto [9] studied some existence results for boundary value problem involving a nonlinear integrodifferential equation of fractional order with integral equation. 
Very recently N'Guérékata [10] discussed the existence of solutions of fractional abstract differential equations with nonlocal initial condition. Anguraj et al. [11] studied the existence and uniqueness theorem for the nonlinear fractional mixed Volterra-Fredholm integrodifferential equation with nonlocal initial condition.

Motivated by these works, we study in this paper the existence of solution of boundary value problem for fractional integrodifferential equations (in the case $1<\alpha \leq 2$ ) in Banach spaces by using Banach and Krasnosel'skii fixed-point theorems.

\section{Preliminaries}

First of all, we recall some basic definitions; see [12-15].

Definition 2.1. For a function $f$ given on the interval $[a, b]$, the Caputo fractional order derivative of $f$ is defined by

$$
{ }_{a}^{t} D^{\alpha} f(t)=\frac{1}{\Gamma(n-\alpha)} \int_{a}^{t}(t-s)^{n-\alpha-1} f^{(n)}(s) d s,
$$

where $n=[\alpha]+1$ and $[\alpha]$ denotes the integer part of $\alpha$.

Lemma 2.2. Let $\alpha>0$, then

$$
{ }_{a}^{t} D^{-\alpha}{ }_{a}^{t} D^{\alpha} y(t)=y(t)+c_{0}+c_{1} t+c_{2} t^{2}+\cdots+c_{n-1} t^{n-1},
$$

for some $c_{i} \in R, i=0,1, \ldots, n-1, n=[\alpha]+1$.

Definition 2.3. Let $f$ be a function which is defined almost everywhere (a.e) on $[a, b]$, for $\alpha>0$, we define

$$
{ }_{a}^{b} D^{-\alpha} f=\frac{1}{\Gamma(\alpha)} \int_{a}^{b}(b-t)^{\alpha-1} f(t) d t
$$

provided that the integral (Lebesgue) exists.

Theorem 2.4 (Krasnosel'skii fixed point theorem). Let $M$ be a closed-convex bounded nonempty subset of a Banach space X. Let $A$ and $B$ be two operators such that

(i) $A x+B y=M$, whenever $x, y \in M$,

(ii) $A$ is compact and continuous;

(iii) $B$ is a contraction mapping,

then there exists $z \in M$ such that $z=A z+B z$.

Let $X$ be a Banach space with the norm $\|\cdot\|$. Let $C=([0, T], X)$ be Banach space ofall 
continuous functions $\psi:[0, T] \rightarrow X$, with supermum norm $\|\psi\|=\sup \{\|\psi(s)\|: s \in$ $[0, T]\}$. Consider the fractional mixed Volttera-Fredholm integrodifferential equation with boundary conditions, which has the form

$$
\begin{gathered}
D^{\alpha} y(t)=f\left(t, y(t), \int_{0}^{t} k(t, s, y(s)) d s, \int_{0}^{T} h_{1}(t, s, y(s)) d s\right), \\
y(0)-y^{\prime}(0)=\int_{0}^{T} g(y(s)) d s, \quad y(T)-y^{\prime}(T)=\int_{0}^{T} h(y(s)) d s,
\end{gathered}
$$

where $1<\alpha \leq 2, D^{\alpha}$ is the Caputo fractional derivative and the nonlinear functions $f:[0, T] \times X \times$ $X \times X \rightarrow X, k, h_{1}:[0, T] \times[0, T] \times X \rightarrow X$ and $g, h: X \rightarrow X$ satisfy the following hypotheses:

(H1) there exists constants $G_{1}, G_{2}$ such that $\|h(y)\| \leq G_{1},\|g(y)\| \leq G_{2}$ for $y \in X$,

(H2) there exists constants $b_{1}, b_{2}$ such that $\|h(x)-h(y)\| \leq b_{1}\|x-y\|$ and

$$
\|g(x)-g(y)\| \leq b_{2}\|x-y\|, \quad \forall x, y \in X,
$$

(H3) there exists continuous functions $p:[0, T] \rightarrow R^{+}=[0, \infty)$ and $p_{1}:[0, T] \rightarrow R^{+}$such that $\left\|\int_{0}^{t}(k(t, s, x)-k(t, s, y)) d s\right\| \leq p(t)\|x-y\|$ and $\left\|\int_{0}^{t} k(t, s, y) d s\right\| \leq p_{1}(t)\|y\|$, for every $t, s \in[0, T]$ and $x, y \in X$,

(H4) there exists continuous functions $q:[0, T] \rightarrow R^{+}$and $q_{1}:[0, T] \rightarrow R^{+}$ such that $\| \int_{0}^{T}\left(h_{1}(t, s, x)-h_{1}(t, s, y) d s\|\leq q(t)\| x-y \|\right.$ and $\left\|\int_{0}^{T} h_{1}(t, s, y) d s\right\| \leq$ $q_{1}(t)\|y\|$ for every $t, s \in[0, T]$ and $x, y \in X$

(H5) there exists continuous function $L:[0, T] \rightarrow R^{+}$, and $N_{1}$ is positive constant such that $\left\|f\left(t, x_{1}, y_{1}, z_{1}\right)-f\left(t, x_{2}, y_{2}, z_{2}\right)\right\| \leq L(t) K\left(\left\|x_{1}-x_{2}\right\|+\left\|y_{1}-y_{2}\right\|+\left\|z_{1}-z_{2}\right\|\right)$ and $N_{1}=\sup _{t \in[0, T]}\|f(t, 0,0,0)\|$, for every $t \in[0, T]$ and $x_{1}, y_{1}, z_{1}, x_{2}, y_{2}, z_{2} \in X$, where $K: R^{+} \rightarrow(0, \infty)$ is continuous nondecreasing function satisfying $K(\gamma(t) x) \leq \gamma(t) K(x)$, where $\gamma$ is a continuous function $\gamma:[0, T] \rightarrow R^{+}$.

Lemma 2.5. Let $1<\alpha \leq 2$ and $f: J \times X \rightarrow X$, where $J=[0, T]$, be a continuous function, then the solution of fractional differential equation (2.4) with the boundary condition (2.5) is

$$
\begin{aligned}
y(t)= & \frac{(1+t)}{T} \int_{0}^{T} h(y(s)) d s+\left(1-\frac{(1+t)}{T}\right) \int_{0}^{T} g(y(s)) d s \\
& -\frac{(1+t)}{T} \int_{0}^{T} \frac{(T-s)^{\alpha-1}}{\Gamma(\alpha)} f\left(s, y(s), \int_{0}^{s} k(s, \tau, y(\tau)) d \tau, \int_{0}^{T} h_{1}(s, \tau, y(\tau)) d \tau\right) \\
& +\frac{(1+t)}{T} \int_{0}^{T} \frac{(T-s)^{\alpha-2}}{\Gamma(\alpha-1)} f\left(s, y(s), \int_{0}^{s} k(s, \tau, y(\tau)) d \tau, \int_{0}^{T} h_{1}(s, \tau, y(\tau)) d \tau\right) d s \\
& +\int_{0}^{t} \frac{(t-s)^{\alpha-1}}{\Gamma(\alpha)} f\left(s, y(s), \int_{0}^{s} k(s, \tau, y(\tau)) d \tau, \int_{0}^{T} h_{1}(s, \tau, y(\tau)) d \tau\right) d s .
\end{aligned}
$$


Proof. By Lemma 2.2, we reduce the problem (2.4)-(2.5) to an equivalent integral equation

$$
\begin{aligned}
& y(t)={ }_{0}^{t} I^{\alpha} f+C_{1}+C_{2} t \\
& y(t)=\int_{0}^{t} \frac{(t-s)^{\alpha-1}}{\Gamma(\alpha)} f\left(s, y(s), \int_{0}^{s} k(s, \tau, y(\tau)) d \tau, \int_{0}^{T} h_{1}(s, \tau, y(\tau)) d \tau\right) d s+C_{1}+C_{2} t
\end{aligned}
$$

In view of the relations ${ }^{c} D^{\alpha} I^{\alpha} y(t)=y(t)$ and $I^{\alpha} I^{\beta} y(t)=I^{\alpha+\beta} y(t)$, for $\alpha, \beta>0$, we obtain

$$
y^{\prime}(t)=\int_{0}^{t} \frac{(t-s)^{\alpha-2}}{\Gamma(\alpha-1)} f\left(s, y(s), \int_{0}^{s} k(s, \tau, y(\tau)) d \tau, \int_{0}^{T} h_{1}(s, \tau, y(\tau)) d \tau\right) d s+C_{2}
$$

Applying the boundary condition (2.5), we find that

$$
\begin{aligned}
y(0)=C_{1}, \quad y(T)= & \int_{0}^{T} \frac{(T-s)^{\alpha-1}}{\Gamma(\alpha)} f\left(s, y(s), \int_{0}^{s} k(s, \tau, y(\tau)) d \tau, \int_{0}^{T} h_{1}(s, \tau, y(\tau)) d \tau\right) d s \\
& +C_{1}+C_{2} T, \\
y^{\prime}(0)=C_{2}, \quad y^{\prime}(T)= & \int_{0}^{T} \frac{(T-s)^{\alpha-2}}{\Gamma(\alpha-1)} f\left(s, y(s), \int_{0}^{s} k(s, \tau, y(\tau)) d \tau, \int_{0}^{T} h_{1}(s, \tau, y(\tau)) d \tau\right) d s \\
& +C_{2},
\end{aligned}
$$

that is,

$$
\begin{aligned}
C_{2}= & \frac{1}{T} \int_{0}^{T} h(y(s)) d s-\frac{1}{T} \int_{0}^{T} g(y(s)) d s \\
& -\frac{1}{T} \int_{0}^{T} \frac{(T-s)^{\alpha-1}}{\Gamma(\alpha)} f\left(s, y(s), \int_{0}^{s} k(s, \tau, y(\tau)) d \tau, \int_{0}^{T} h_{1}(s, \tau, y(\tau)) d \tau\right) d s \\
& +\frac{1}{T} \int_{0}^{T} \frac{(T-s)^{\alpha-2}}{\Gamma(\alpha-1)} f\left(s, y(s), \int_{0}^{s} k(s, \tau, y(\tau)) d \tau, \int_{0}^{T} h_{1}(s, \tau, y(\tau)) d \tau\right) d s, \\
C_{1}= & \frac{1}{T} \int_{0}^{T} h(y(s)) d s+\left(1-\frac{1}{T}\right) \int_{0}^{T} g(y(s)) d s \\
& -\frac{1}{T} \int_{0}^{T} \frac{(T-s)^{\alpha-1}}{\Gamma(\alpha)} f\left(s, y(s), \int_{0}^{s} k(s, \tau, y(\tau)) d \tau, \int_{0}^{T} h_{1}(s, \tau, y(\tau)) d \tau\right) d s \\
& +\frac{1}{T} \int_{0}^{T} \frac{(T-s)^{\alpha-2}}{\Gamma(\alpha-1)} f\left(s, y(s), \int_{0}^{s} k(s, \tau, y(\tau)) d \tau, \int_{0}^{T} h_{1}(s, \tau, y(\tau)) d \tau\right) d s .
\end{aligned}
$$


Therefore the solution of (2.4)-(2.5) is

$$
\begin{aligned}
y(t)= & \frac{(1+t)}{T} \int_{0}^{T} h(y(s)) d s+\left(1-\frac{(1+t)}{T}\right) \int_{0}^{T} g(y(s)) d s \\
& -\frac{(1+t)}{T} \int_{0}^{T} \frac{(T-s)^{\alpha-1}}{\Gamma(\alpha)} f\left(s, y(s), \int_{0}^{s} k(s, \tau, y(\tau)) d \tau, \int_{0}^{T} h_{1}(s, \tau, y(\tau)) d \tau\right) d s \\
& +\frac{(1+t)}{T} \int_{0}^{T} \frac{(T-s)^{\alpha-2}}{\Gamma(\alpha-1)} f\left(s, y(s), \int_{0}^{s} k(s, \tau, y(\tau)) d \tau, \int_{0}^{T} h_{1}(s, \tau, y(\tau)) d \tau\right) d s \\
& +\int_{0}^{t} \frac{(t-s)^{\alpha-1}}{\Gamma(\alpha)} f\left(s, y(s), \int_{0}^{s} k(s, \tau, y(\tau)) d \tau, \int_{0}^{T} h_{1}(s, \tau, y(\tau)) d \tau\right) d s,
\end{aligned}
$$

which completes the proof.

\section{The Main Result}

Theorem 3.1. If the hypotheses (H1)-(H5) are satisfied, then the fractional integrodifferential equation (2.4)-(2.5) has a unique solution on $\mathrm{J}$.

Proof. Define $F: C \rightarrow C$ by

$$
\begin{aligned}
F y(t)= & \frac{(1+t)}{T} \int_{0}^{T} h(y(s)) d s+\left(1-\frac{(1+t)}{T}\right) \int_{0}^{T} g(y(s)) d s \\
& -\frac{(1+t)}{T} \int_{0}^{T} \frac{(T-s)^{\alpha-1}}{\Gamma(\alpha)} f\left(s, y(s), \int_{0}^{s} k(s, \tau, y(\tau)) d \tau, \int_{0}^{T} h_{1}(s, \tau, y(\tau)) d \tau\right) d s \\
& +\frac{(1+t)}{T} \int_{0}^{T} \frac{(T-s)^{\alpha-2}}{\Gamma(\alpha-1)} f\left(s, y(s), \int_{0}^{s} k(s, \tau, y(\tau)) d \tau, \int_{0}^{T} h_{1}(s, \tau, y(\tau)) d \tau\right) d s \\
& +\int_{0}^{t} \frac{(t-s)^{\alpha-1}}{\Gamma(\alpha)} f\left(s, y(s), \int_{0}^{s} k(s, \tau, y(\tau)) d \tau, \int_{0}^{T} h_{1}(s, \tau, y(\tau)) d \tau\right) d s .
\end{aligned}
$$

We show that $F$ has a fixed point on $B r$. This fixed point is then a solution of (2.4)-(2.5). Firstly, we show that $F B r \subset B r$, where $B r=\{y \in C:\|y\| \leq r\}$. For $y \in B r$, we have

$$
\begin{aligned}
\|F y(t)\| \leq & \frac{(1+t)}{T} \int_{0}^{T}\|h(y(s))\| d s+\left(1-\frac{(1+t)}{T}\right) \int_{0}^{T}\|g(y(s))\| d s \\
& +\frac{(1+t)}{T} \int_{0}^{T} \frac{(T-s)^{\alpha-2}}{\Gamma(\alpha-1)}\left\|f\left(s, y(s), \int_{0}^{s} k(s, \tau, y(\tau)) d \tau, \int_{0}^{T} h_{1}(s, \tau, y(\tau)) d \tau\right)\right\| d s
\end{aligned}
$$




$$
\begin{aligned}
& +\frac{(1+t)}{T} \int_{0}^{T} \frac{(T-s)^{\alpha-1}}{\Gamma(\alpha)}\left\|f\left(s, y(s), \int_{0}^{s} k(s, \tau, y(\tau)) d \tau, \int_{0}^{T} h_{1}(s, \tau, y(\tau)) d \tau\right)\right\| d s \\
& +\int_{0}^{t} \frac{(t-s)^{\alpha-1}}{\Gamma(\alpha)}\left\|f\left(s, y(s), \int_{0}^{s} k(s, \tau, y(\tau)) d \tau, \int_{0}^{T} h_{1}(s, \tau, y(\tau)) d \tau\right)\right\| d s,
\end{aligned}
$$

$\|F y(t)\| \leq \frac{(1+t)}{T} \int_{0}^{T}\|h(y(s))\| d s+\left(1-\frac{(1+t)}{T}\right) \int_{0}^{T}\|g(y(s))\| d s$

$$
\begin{gathered}
+\frac{(1+t)}{T} \int_{0}^{T} \frac{(T-s)^{\alpha-2}}{\Gamma(\alpha-1)} \| f\left(s, y(s), \int_{0}^{S} k(s, \tau, y(\tau)) d \tau, \int_{0}^{T} h_{1}(s, \tau, y(\tau)) d \tau\right) \\
-f(s, 0,0,0)+f(s, 0,0,0) \| d s \\
+\frac{(1+t)}{T} \int_{0}^{T} \frac{(T-s)^{\alpha-1}}{\Gamma(\alpha)} \| f\left(s, y(s), \int_{0}^{S} k(s, \tau, y(\tau)) d \tau, \int_{0}^{T} h_{1}(s, \tau, y(\tau)) d \tau\right) \\
\left.+\int_{0}^{t} \frac{(t-s)^{\alpha-1}}{\Gamma(\alpha)}\|f(s, y, 0,0)+f(s, 0,0,0)\| d s, \int_{0}^{S} k(s, \tau, y(\tau)) d \tau, \int_{0}^{T} h_{1}(s, \tau, y(\tau)) d \tau\right) \\
-f(s, 0,0,0)+f(s, 0,0,0) \| d s,
\end{gathered}
$$

$\|F y(t)\| \leq \frac{(1+t)}{T} G_{1} T+\left(1-\frac{(1+t)}{T}\right) G_{2} T$

$$
\begin{aligned}
& +\frac{(1+t)}{T} \int_{0}^{T} \frac{(T-s)^{\alpha-2}}{\Gamma(\alpha-1)} \| f\left(s, y(s), \int_{0}^{s} k(s, \tau, y(\tau)) d \tau, \int_{0}^{T} h_{1}(s, \tau, y(\tau)) d \tau\right) \\
& -f(s, 0,0,0)\left\|d s+\frac{(1+t)}{T} \int_{0}^{T} \frac{(T-s)^{\alpha-2}}{\Gamma(\alpha-1)}\right\| f(s, 0,0,0) \| d s \\
& +\int_{0}^{t} \frac{(t-s)^{\alpha-1}}{\Gamma(\alpha)} \| f\left(s, y(s), \int_{0}^{S} k(s, \tau, y(\tau)) d \tau, \int_{0}^{T} h_{1}(s, \tau, y(\tau)) d \tau\right) \\
& +\left(\frac{1+t}{T}\right) \int_{0}^{T} \frac{(T-s)^{\alpha-1}}{\Gamma(\alpha)} \| f\left(s, y(s), \int_{0}^{S} k(s, \tau, y(\tau)) d \tau, \int_{0}^{T} h_{1}(s, \tau, y(\tau)) d \tau\right) \\
& \quad-f(s, 0,0,0)\left\|d s+\left(\frac{1+t}{T}\right) \int_{0}^{T} \frac{(T-s)^{\alpha-1}}{\Gamma(\alpha)}\right\| f(s, 0,0,0) \| d s \\
& \leq \frac{(1+t)}{T} G_{1} T+\left(1-\frac{(1+t)}{T}\right) G_{2} T+\frac{(1-s)^{\alpha-1}}{\Gamma(\alpha)}\|f(s, 0,0,0)\| d s \\
& T
\end{aligned}
$$


International Journal of Differential Equations

$$
\begin{aligned}
& \left.K\left(\|y(s)\|+\left\|\int_{0}^{S} k(s, \tau, y(\tau)) d \tau\right\|+\| \int_{0}^{T} h_{1}(s, \tau, y(\tau)) d \tau\right) \|\right) d s \\
& +\frac{(1+t) N_{1}}{T} \int_{0}^{T} \frac{(T-s)^{\alpha-2}}{\Gamma(\alpha-1)} d s+\frac{(1+t)}{T} \int_{0}^{T} \frac{(T-s)^{\alpha-1}}{\Gamma(\alpha)} L(s) \\
& \left.K\left(\|y(s)\|+\left\|\int_{0}^{s} k(s, \tau, y(\tau)) d \tau\right\|+\| \int_{0}^{T} h_{1}(s, \tau, y(\tau)) d \tau\right) \|\right) d s \\
& +\frac{(1+t) N_{1}}{T} \int_{0}^{T} \frac{(T-s)^{\alpha-1}}{\Gamma(\alpha)} d s+\int_{0}^{t} \frac{(t-s)^{\alpha-1}}{\Gamma(\alpha)} L(s) \\
& \left.K\left(\|y(s)\|+\left\|\int_{0}^{s} k(s, \tau, y(\tau)) d \tau\right\|+\| \int_{0}^{T} h_{1}(s, \tau, y(\tau)) d \tau\right) \|\right) d s \\
& +N_{1} \int_{0}^{t} \frac{(t-s)^{\alpha-1}}{\Gamma(\alpha)} d s \\
& \leq(1+t) G_{1}+\left(1-\frac{(1+t)}{T}\right) G_{2} T+\frac{(1+t) N_{1}}{T} \\
& \left(\int_{0}^{T} \frac{(T-s)^{\alpha-2}}{\Gamma(\alpha-1)} d s+\int_{0}^{T} \frac{(T-s)^{\alpha-1}}{\Gamma(\alpha)} d s\right)+N_{1} \int_{0}^{t} \frac{(t-s)^{\alpha-1}}{\Gamma(\alpha)} d s \\
& +\frac{(1+t)}{T} \int_{0}^{T} \frac{(T-s)^{\alpha-2}}{\Gamma(\alpha-1)} L(s) K\left(\|y\|+p_{1}(s)\|y\|+q_{1}(s)\|y\|\right) d s \\
& +\frac{(1+t)}{T} \int_{0}^{T} \frac{(T-s)^{\alpha-1}}{\Gamma(\alpha)} L(s) K\left(\|y\|+p_{1}(s)\|y\|+q_{1}(s)\|y\|\right) d s \\
& +\int_{0}^{t} \frac{(t-s)^{\alpha-1}}{\Gamma(\alpha)} L(s) K\left(\|y\|+p_{1}(s)\|y\|+q_{1}(s)\|y\|\right) d s,
\end{aligned}
$$

$\|F y(t)\| \leq(1+t) G_{1}+\left(1-\frac{(1+t)}{T}\right) G_{2} T$

$$
\begin{aligned}
& +\frac{(1+t) N_{1}}{T}\left(\frac{T^{\alpha-1}}{\Gamma(\alpha)}+\frac{T^{\alpha}}{\Gamma(\alpha+1)}\right)+N_{1} \frac{T^{\alpha}}{\Gamma(\alpha+1)} \\
& +\frac{(1+t)}{T} \int_{0}^{T} \frac{(T-s)^{\alpha-2}}{\Gamma(\alpha-1)} L(s)\left(1+p_{1}(s)+q_{1}(s)\right) K(\|y\|) d s \\
& +\frac{(1+t)}{T} \int_{0}^{T} \frac{(T-s)^{\alpha-1}}{\Gamma(\alpha)} L(s)\left(1+p_{1}(s)+q_{1}(s)\right) K(\|y\|) d s \\
& +\int_{0}^{t} \frac{(t-s)^{\alpha-1}}{\Gamma(\alpha)} L(s)\left(1+p_{1}(s)+q_{1}(s)\right) K(\|y\|) d s .
\end{aligned}
$$


Since we have $M_{1}=\sup \left\{L(t)\left(1+p_{1}(t)+q_{1}(t)\right) ; t \in[0, T]\right\}$, and $(1-((1+t) / T))<(1-(1 / T))$, we get

$$
\begin{aligned}
& \leq(1+t) G_{1}+\left(1-\frac{1}{T}\right) G_{2} T+\frac{(1+t) N_{1}}{T}\left(\frac{T^{\alpha-1}}{\Gamma(\alpha)}+\frac{T^{\alpha}}{\Gamma(\alpha+1)}\right)+N_{1} \frac{T^{\alpha}}{\Gamma(\alpha+1)} \\
& +\frac{(1+t) M_{1}}{T} \int_{0}^{T} \frac{(T-s)^{\alpha-2}}{\Gamma(\alpha-1)} K(\|y\|) d s+\frac{(1+t) M_{1}}{T} \int_{0}^{T} \frac{(T-s)^{\alpha-1}}{\Gamma(\alpha)} K(\|y\|) d s \\
& +M_{1} \int_{0}^{t} \frac{(t-s)^{\alpha-1}}{\Gamma(\alpha)} K(\|y\|) d s \\
& \leq(1+t) G_{1}+\left(1-\frac{1}{T}\right) G_{2} T+\frac{(1+t) N_{1}}{T}\left(\frac{T^{\alpha-1}}{\Gamma(\alpha)}+\frac{T^{\alpha}}{\Gamma(\alpha+1)}\right)+N_{1} \frac{T^{\alpha}}{\Gamma(\alpha+1)} \\
& +\frac{(1+t) M_{1} K(r)}{T} \int_{0}^{T} \frac{(T-s)^{\alpha-2}}{\Gamma(\alpha-1)} d s+\frac{(1+t) M_{1} K(r)}{T} \int_{0}^{T} \frac{(T-s)^{\alpha-1}}{\Gamma(\alpha)} d s \\
& +M_{1} K(r) \int_{0}^{t} \frac{(t-s)^{\alpha-1}}{\Gamma(\alpha)} d s \\
& \leq(1+t) G_{1}+\left(1-\frac{1}{T}\right) G_{2} T+\frac{(1+t) N_{1}}{T}\left(\frac{T^{\alpha-1}}{\Gamma(\alpha)}+\frac{T^{\alpha}}{\Gamma(\alpha+1)}\right)+N_{1} \frac{T^{\alpha}}{\Gamma(\alpha+1)} \\
& +\frac{(1+t) M_{1} K(r)}{T}\left(\frac{T^{\alpha-1}}{\Gamma(\alpha)}+\frac{T^{\alpha}}{\Gamma(\alpha+1)}\right)+\frac{M_{1} K(r) T^{\alpha}}{\Gamma(\alpha+1)} \\
& \leq(1+t) G_{1}+(T-1) G_{2}+\frac{(1+t)}{T}\left(N_{1}+M_{1} K(r)\right)\left(\frac{T^{\alpha-1}}{\Gamma(\alpha)}+\frac{T^{\alpha}}{\Gamma(\alpha+1)}\right) \\
& +\left(N_{1}+M_{1} K(r)\right) \frac{T^{\alpha}}{\Gamma(\alpha+1)}, \\
& \|F y(t)\| \leq(1+T) G_{1}+(T-1) G_{2}+\frac{(1+T)}{T}\left(N_{1}+M_{1} K(r)\right)\left(\frac{T^{\alpha-1}}{\Gamma(\alpha)}+\frac{T^{\alpha}}{\Gamma(\alpha+1)}\right) \\
& +\left(N_{1}+M_{1} K(r)\right) \frac{T^{\alpha}}{\Gamma(\alpha+1)} \\
& \leq G_{1}(1+T)+G_{2}(T-1)+\frac{C_{0}\left(N_{1}+M_{1} K(r)\right)}{\Gamma(\alpha+1) T^{2-\alpha}},
\end{aligned}
$$

where $C_{0}=2 T^{2}+T+\alpha(T+1)$.

Now, take $x, y \in C$ and for each $t \in[0, T]$, we obtain

$$
\begin{aligned}
\|F x(t)-F y(t)\| \leq & \frac{(1+t)}{T} \int_{0}^{T}\|h(x)-h(y)\| d s+\left(1-\frac{(1+t)}{T}\right) \int_{0}^{T}\|g(x)-g(y)\| d s \\
& +\frac{(1+t)}{T} \int_{0}^{T} \frac{(T-s)^{\alpha-1}}{\Gamma(\alpha)}
\end{aligned}
$$


International Journal of Differential Equations

$$
\begin{aligned}
& \| f\left(s, x(s), \int_{0}^{s} k(s, \tau, x(\tau)) d \tau, \int_{0}^{T} h_{1}(s, \tau, x(\tau)) d \tau\right) \\
& \quad-f\left(s, y(s), \int_{0}^{s} k(s, \tau, y(\tau)) d \tau, \int_{0}^{T} h_{1}(s, \tau, y(\tau)) d \tau\right) \| d s \\
& +\frac{(1+t)}{T} \int_{0}^{T} \frac{(T-s)^{\alpha-2}}{\Gamma(\alpha-1)} \\
& \| f\left(s, x(s), \int_{0}^{s} k(s, \tau, x(\tau)) d \tau, \int_{0}^{T} h_{1}(s, \tau, x(\tau)) d \tau\right) \\
& \quad-f\left(s, y(s), \int_{0}^{s} k(s, \tau, y(\tau)) d \tau, \int_{0}^{T} h_{1}(s, \tau, y(\tau)) d \tau\right) \| d s \\
& +\int_{0}^{t} \frac{(t-s)^{\alpha-1}}{\Gamma(\alpha)} \\
& \| f\left(s, x(s), \int_{0}^{s} k(s, \tau, x(\tau)) d \tau, \int_{0}^{T} h_{1}(s, \tau, x(\tau)) d \tau\right) \\
& \quad-f\left(s, y(s), \int_{0}^{s} k(s, \tau, y(\tau)) d \tau, \int_{0}^{T} h_{1}(s, \tau, y(\tau)) d \tau\right) \| d s,
\end{aligned}
$$

by using (H1)-(H5), we get

$$
\begin{aligned}
\|F x(t)-F y(t)\| \leq & \frac{b_{1}(1+t)}{T} \int_{0}^{T}\|x-y\| d s+b_{2}\left(1-\frac{(1+t)}{T}\right) \int_{0}^{T}\|x-y\| d s \\
& +\frac{(1+t)}{T} \int_{0}^{T} \frac{(T-s)^{\alpha-2}}{\Gamma(\alpha-1)} L(s) \\
& K\left(\|x(s)-y(s)\|+\left\|\int_{0}^{s}(k(s, \tau, x(\tau))-k(s, \tau, y(\tau))) d \tau\right\|\right. \\
& \left.+\left\|\int_{0}^{T}\left(h_{1}(s, \tau, x(\tau))-h_{1}(s, \tau, y(\tau))\right) d \tau\right\|\right) d s \\
& +\frac{(1+t)}{T} \int_{0}^{T} \frac{(T-s)^{\alpha-1}}{\Gamma(\alpha)} L(s) \\
& K\left(\|x(s)-y(s)\|+\left\|\int_{0}^{s}(k(s, \tau, x(\tau))-k(s, \tau, y(\tau))) d \tau\right\|\right. \\
& \left.+\left\|\int_{0}^{T}\left(h_{1}(s, \tau, x(\tau))-h_{1}(s, \tau, y(\tau))\right) d \tau\right\|\right) d s+\int_{0}^{t} \frac{(t-s)^{\alpha-1}}{\Gamma(\alpha)} L(s)
\end{aligned}
$$




$$
\begin{aligned}
& K\left(\|x(s)-y(s)\|+\left\|\int_{0}^{s}(k(s, \tau, x(\tau))-k(s, \tau, y(\tau))) d \tau\right\|\right. \\
& \left.+\left\|\int_{0}^{T}\left(h_{1}(s, \tau, x(\tau))-h_{1}(s, \tau, y(\tau))\right) d \tau\right\|\right) d s \\
& \leq \frac{b_{1}(1+t)}{T} \int_{0}^{T}\|x-y\| d s+b_{2}\left(1-\frac{(1+t)}{T}\right) \int_{0}^{T}\|x-y\| d s \\
& +\frac{(1+t)}{T} \int_{0}^{T} \frac{(T-s)^{\alpha-2}}{\Gamma(\alpha-1)} L(s) K(\|x-y\|+p(s)\|x-y\|+q(s)\|x-y\|) d s \\
& +\frac{(1+t)}{T} \int_{0}^{T} \frac{(T-s)^{\alpha-1}}{\Gamma(\alpha)} L(s) K(\|x-y\|+p(s)\|x-y\|+q(s)\|x-y\|) d s \\
& +\int_{0}^{t} \frac{(t-s)^{\alpha-1}}{\Gamma(\alpha)} L(s) K(\|x-y\|+p(s)\|x-y\|+q(s)\|x-y\|) d s \\
& \leq \frac{b_{1}(1+t)}{T} \int_{0}^{T}\|x-y\| d s+b_{2}\left(1-\frac{1}{T}\right) \int_{0}^{T}\|x-y\| d s \\
& +\frac{(1+t)}{T} \int_{0}^{T} \frac{(T-s)^{\alpha-2}}{\Gamma(\alpha-1)} L(s)(1+p(s)+q(s)) K(\|x-y\|) d s \\
& +\frac{(1+t)}{T} \int_{0}^{T} \frac{(T-s)^{\alpha-1}}{\Gamma(\alpha)} L(s)(1+p(s)+q(s)) K(\|x-y\|) d s \\
& +\int_{0}^{t} \frac{(t-s)^{\alpha-1}}{\Gamma(\alpha)} L(s)(1+p(s)+q(s)) K(\|x-y\|) d s .
\end{aligned}
$$

Since we have $M(t)=L(t)\left(1+p(t)+q(t), M^{*}=\sup \{M(t): t \in[0, T]\}\right.$, and, Let $K(\|x-y\|) \leq$ $w\|x-y\|,(w>0)$, then

$$
\begin{aligned}
\|F x(t)-F y(t)\| \leq & \frac{b_{1}(1+t)}{T} \int_{0}^{T}\|x-y\| d s+b_{2}\left(1-\frac{1}{T}\right) \int_{0}^{T}\|x-y\| d s \\
& +\frac{w M^{*}(1+t)}{T} \int_{0}^{T} \frac{(T-s)^{\alpha-2}}{\Gamma(\alpha-1)}\|x-y\| d s \\
& +\frac{w M^{*}(1+t)}{T} \int_{0}^{T} \frac{(T-s)^{\alpha-1}}{\Gamma(\alpha)}\|x-y\| d s \\
& +w M^{*} \int_{0}^{t} \frac{(t-s)^{\alpha-1}}{\Gamma(\alpha)}\|x-y\| d s \\
\leq & {\left[b_{1}(1+T)+b_{2}(T-1)+\frac{w M^{*} C_{1}(1+T)}{\Gamma(\alpha+1) T^{2-\alpha}}\right]\|x-y\|, }
\end{aligned}
$$


where $C_{1}=2 T^{2}+T+\alpha(1+T)$.

As $b_{1}(1+T)+b_{2}(T-1)+\left(w M^{*} C_{1}(1+T)\right) /\left(\Gamma(\alpha+1) T^{2-\alpha}\right)<1$, therefore $f$ is a contraction. Thus, the conclusion of the theorem is followed by the contraction mapping principle.

Theorem 3.2. Assume that (H1)-(H5) hold with

$$
\left\|f\left(t, y(t), \int_{0}^{t} k(t, \tau, y(\tau)) d \tau, \int_{0}^{T} h_{1}(t, \tau, y(\tau)) d \tau\right)\right\| \leq \psi(t), \quad \text { where } \psi(t) \in L_{1}(J) .
$$

Then the boundary value problem (2.4)-(2.5) has at least one element on $[0, T]$.

Proof. Consider $B r=\{y \in C:\|y\| \leq r\}$. We define the operators $A$ and $B$ as

$$
\begin{aligned}
(A x)(t)= & \frac{1}{\Gamma(\alpha)} \int_{0}^{t}(t-s)^{\alpha-1} f\left(t, x(s), \int_{0}^{s} k(s, \tau, x(\tau)) d \tau, \int_{0}^{T} h_{1}(s, \tau, x(\tau)) d \tau\right) d s \\
(B x)(t)= & \frac{(1+t)}{T} \int_{0}^{T} h(y(s)) d s+\left(1-\frac{(1+t)}{T}\right) \int_{0}^{T} g(y(s)) d s+\frac{(1+t)}{T} \int_{0}^{T} \frac{(T-s)^{\alpha-2}}{\Gamma(\alpha-1)} \\
& f\left(s, x(s), \int_{0}^{s} k(s, \tau, x(\tau)) d \tau, \int_{0}^{T} h_{1}(s, \tau, x(\tau)) d \tau\right) d s \\
& +\frac{(1+t)}{T} \int_{0}^{T} \frac{(T-s)^{\alpha-1}}{\Gamma(\alpha)} f\left(s, x(s), \int_{0}^{s} k(s, \tau, x(\tau)) d \tau, \int_{0}^{T} h_{1}(s, \tau, x(\tau)) d \tau\right) d s
\end{aligned}
$$

Let us observe that if $x, y \in B r$, then $A x+B y \in B r$,

$$
\begin{aligned}
\|A x+B y\|=\| & \int_{0}^{t} \frac{(t-s)^{\alpha-1}}{\Gamma(\alpha)} f\left(s, x(s), \int_{0}^{s} k(s, \tau, x(\tau)) d \tau, \int_{0}^{T} h_{1}(s, \tau, x(\tau)) d \tau\right) d s \\
& +\frac{(1+t)}{T} \int_{0}^{T} h(y(s)) d s+\left(1-\frac{(1+t)}{T}\right) \int_{0}^{T} g(y(s)) d s+\frac{(1+t)}{T} \int_{0}^{T} \frac{(T-s)^{\alpha-2}}{\Gamma(\alpha-1)} \\
& f\left(s, y(s), \int_{0}^{s} k(s, \tau, y(\tau)) d \tau, \int_{0}^{T} h_{1}(s, \tau, y(\tau)) d \tau\right) d s+\frac{(1+t)}{T} \\
& \int_{0}^{T} \frac{(T-s)^{\alpha-1}}{\Gamma(\alpha)} f\left(s, y(s), \int_{0}^{s} k(s, \tau, y(\tau)) d \tau, \int_{0}^{T} h_{1}(s, \tau, y(\tau)) d \tau\right) d s \| \\
\leq & \int_{0}^{t} \frac{(t-s)^{\alpha-1}}{\Gamma(\alpha)}\left\|f\left(s, x(s), \int_{0}^{s} k(s, \tau, x(\tau)) d \tau, \int_{0}^{T} h_{1}(s, \tau, x(\tau)) d \tau\right)\right\| d s
\end{aligned}
$$




$$
\begin{aligned}
& +\frac{(1+t)}{T} \int_{0}^{T}\|h(y(s))\| d s+\left(1-\frac{(1+t)}{T}\right) \int_{0}^{T}\|g(y(s))\| d s \\
& +\frac{(1+t)}{T} \int_{0}^{T} \frac{(T-s)^{\alpha-2}}{\Gamma(\alpha-1)} \\
& \left\|f\left(s, y(s), \int_{0}^{s} k(s, \tau, y(\tau)) d \tau, \int_{0}^{T} h_{1}(s, \tau, y(\tau)) d \tau\right)\right\| d s \\
& +\frac{(1+t)}{T} \int_{0}^{T} \frac{(T-s)^{\alpha-1}}{\Gamma(\alpha)} \\
& \left\|f\left(s, y(s), \int_{0}^{s} k(s, \tau, y(\tau)) d \tau, \int_{0}^{T} h_{1}(s, \tau, y(\tau)) d \tau\right)\right\| d s \\
& \leq\|\psi\|_{L_{1}} \int_{0}^{t} \frac{(t-s)^{\alpha-1}}{\Gamma(\alpha)} d s+(1+t) G_{1} \\
& +\left(1-\frac{(1+t)}{T}\right) G_{2} T+\frac{(1+t)\|\psi\|_{L_{1}}}{T} \int_{0}^{T} \frac{(T-s)^{\alpha-2}}{\Gamma(\alpha-1)} d s \\
& +\frac{(1+t)\|\psi\|_{L_{1}}}{T} \int_{0}^{T} \frac{(T-s)^{\alpha-1}}{\Gamma(\alpha)} d s \\
& \leq \frac{\|\psi\|_{L_{1}} T^{\alpha}}{\Gamma(\alpha+1)}+(1+t) G_{1}+\left(1-\frac{1}{T}\right) G_{2} T+\frac{(1+t) T^{\alpha-1}\|\psi\|_{L_{1}}}{T \Gamma(\alpha)}+\frac{(1+t) T^{\alpha}\|\psi\|_{L_{1}}}{T \Gamma(\alpha+1)} \\
& \leq \frac{\|\psi\|_{L_{1}} T^{\alpha}}{\Gamma(\alpha+1)}+\frac{(1+t) T^{\alpha-1}\|\psi\|_{L_{1}}}{T \Gamma(\alpha)}+\frac{(1+t) T^{\alpha}\|\psi\|_{L_{1}}}{T \Gamma(\alpha+1)}+(1+T) G_{1}+(T-1) G_{2} \\
& \leq G_{1}(1+T)+G_{2}(T-1)+\frac{C_{2} T^{\alpha-2}}{\Gamma(\alpha+1)}\|\psi\|_{L_{1}}
\end{aligned}
$$

where $C_{2}=2 T^{2}+T(\alpha+1)+T$.

Now we prove that $B x$ is contraction mapping,

$$
\begin{aligned}
\left\|B x_{1}-B x_{2}\right\| \leq & \frac{(1+t)}{T} \int_{0}^{T} \frac{(T-s)^{\alpha-1}}{\Gamma(\alpha)} \| f\left(s, x_{1}(s), \int_{0}^{s} k\left(s, \tau, x_{1}(\tau)\right) d \tau, \int_{0}^{T} h_{1}\left(s, \tau, x_{1}(\tau)\right) d \tau\right) \\
& -f\left(s, x_{2}(s) \int_{0}^{s} k\left(s, \tau, x_{2}(\tau)\right) d \tau, \int_{0}^{T} h_{1}\left(s, \tau, x_{2}(\tau)\right) d \tau\right) \| d s \\
+ & \frac{(1+t)}{T} \int_{0}^{T} \frac{(T-s)^{\alpha-2}}{\Gamma(\alpha-1)} \| f\left(s, x_{1}(s), \int_{0}^{s} k\left(s, \tau, x_{1}(\tau)\right) d \tau, \int_{0}^{T} h_{1}\left(s, \tau, x_{1}(\tau)\right) d \tau\right) \\
& -f\left(s, x_{2}(s), \int_{0}^{s} k\left(s, \tau, x_{2}(\tau)\right) d \tau, \int_{0}^{T} h_{1}\left(s, \tau, x_{2}(\tau)\right) d \tau\right) \| d s
\end{aligned}
$$




$$
\begin{aligned}
\leq \frac{(1+t)}{T}\left[\int_{0}^{T} \frac{(T-s)^{\alpha-2}}{\Gamma(\alpha-1)} L(s)(1+p(s)+q(s)) K\left(\left\|x_{1}-x_{2}\right\|\right) d s\right. \\
\left.\quad+\int_{0}^{T} \frac{(T-s)^{\alpha-1}}{\Gamma(\alpha)} L(s)(1+p(s)+q(s)) K\left(\left\|x_{1}-x_{2}\right\|\right) d s\right] .
\end{aligned}
$$

Let $K\left(\left\|x_{1}-x_{2}\right\|\right) \leq w\left\|x_{1}-x_{2}\right\|$, we obtain

$$
\begin{aligned}
\left\|B x_{1}-B x_{2}\right\| & \leq \frac{(1+t) w M^{*}}{T}\left\|x_{1}-x_{2}\right\|\left[\int_{0}^{T} \frac{(T-s)^{\alpha-2}}{\Gamma(\alpha-1)} d s+\int_{0}^{T} \frac{(T-s)^{\alpha-1}}{\Gamma(\alpha)} d s\right], \\
\left\|B x_{1}-B x_{2}\right\| & \leq \frac{(1+T) w M^{*}}{T}\left[\frac{T^{\alpha-1}}{\Gamma(\alpha)}+\frac{T^{\alpha}}{\Gamma(\alpha+1)}\right]\left\|x_{1}-x_{2}\right\| \\
& \leq \frac{w M^{*}(1+T)(\alpha+T)}{\Gamma(\alpha+1) T^{2-\alpha}}\left\|x_{1}-x_{2}\right\| .
\end{aligned}
$$

It is clear that $B$ is contraction mapping, since $x(t)$ is continuous, then $A x$ is continuous

$$
\begin{aligned}
\|A x(t)\| & =\left\|\frac{1}{\Gamma(\alpha)} \int_{0}^{t}(t-s)^{\alpha-1} f\left(s, x(s), \int_{0}^{s} k(s, \tau, x(\tau)) d \tau, \int_{0}^{T} h_{1}(s, \tau, x(\tau)) d \tau\right) d s\right\| \\
& \leq\|\psi\|_{L_{1}} \int_{0}^{t} \frac{(t-s)^{\alpha-1}}{\Gamma(\alpha)} d s \\
\|A x(t)\| & \leq \frac{T^{\alpha}\|\psi\|_{L_{1}}}{\Gamma(\alpha+1)} .
\end{aligned}
$$

Hence, $A$ is uniformly bounded on $B r$. Now, let us prove that $A x(t)$ is equicontinuous, let $t_{1}, t_{2} \in[0, T]$ and $x \in B r$. Using the fact that $f$ is bounded on the compact set $J \times B r$, thus $\sup _{(t, s) \in J \times B r}\left\|f\left(s, x(s), \int_{0}^{s} k(s, \tau, x(\tau)) d \tau, \int_{0}^{T} h_{1}(s, \tau, x(\tau)) d \tau\right)\right\|=c_{0}<\infty$, we get

$$
\begin{aligned}
\left\|A x\left(t_{1}\right)-A x\left(t_{2}\right)\right\|= & \| \frac{1}{\Gamma(\alpha)} \int_{0}^{t_{1}}\left(t_{1}-s\right)^{\alpha-1} f\left(s, x(s), \int_{0}^{s} k(s, \tau, x(\tau)) d \tau,\right. \\
& \left.\int_{0}^{T} h_{1}(s, \tau, x(\tau)) d \tau\right) d s-\frac{1}{\Gamma(\alpha)} \int_{0}^{t_{2}}\left(t_{2}-s\right)^{\alpha-1} \\
& f\left(s, x(s), \int_{0}^{s} k(s, \tau, x(\tau)) d \tau, \int_{0}^{T} h_{1}(s, \tau, x(\tau)) d \tau\right) d s \| \\
\leq & \frac{1}{\Gamma(\alpha)} \| \int_{0}^{t_{1}}\left[\left(t_{1}-s\right)^{\alpha-1}-\left(t_{2}-s\right)^{\alpha-1}\right]
\end{aligned}
$$




$$
\begin{gathered}
f\left(s, x(s), \int_{0}^{s} k(s, \tau, x(\tau)) d \tau, \int_{0}^{T} h_{1}(s, \tau, x(\tau)) d \tau\right) d s \| \\
+\left\|\int_{t_{1}}^{t_{2}}\left(t_{2}-s\right)^{\alpha-1} f\left(s, x(s), \int_{0}^{s} k(s, \tau, x(\tau)) d \tau, \int_{0}^{T} h_{1}(s, \tau, x(\tau)) d \tau\right) d s\right\| \\
\leq \frac{c_{0}}{\Gamma(\alpha+1)}\left[2\left(t_{2}-t_{1}\right)^{\alpha}+\left(t_{1}{ }^{\alpha}-t_{2}{ }^{\alpha}\right)\right] .
\end{gathered}
$$

So $A$ is relatively compact. By Arzela-Ascoli theorem, $A$ is compact. Now we conclude the result of the theorem of Krasnosel'skii theorem.

Example 3.3. Consider the following fractional mixed Volterra-Fredholm integrodifferential equation:

$$
y^{(1.5)}(t)=\frac{1}{10}+\frac{1}{10+|y(t)|}+\int_{0}^{t} \frac{|y(t)|}{10 e^{|y(t)|}+t} d t+\int_{0}^{1} \frac{|y(t)| e^{-t}}{10+|y(t)|^{2}} d t
$$

with integral boundary conditions

$$
y(0)-y^{\prime}(0)=\int_{0}^{1} \frac{1}{10+|y(t)|} d t, \quad y(1)-y^{\prime}(1)=\int_{0}^{1} \frac{1}{10+e^{-|y(t)|}} d t .
$$

Here,

$$
\begin{array}{r}
\|g(y(t))\|=\left\|\frac{1}{10+|y(t)|}\right\| \leq \frac{1}{10}, \quad\|g(x)-g(y)\| \leq \frac{1}{100}\|x-y\|, \\
\|h(y(t))\|=\left\|\frac{1}{10+e^{-|y(t)|}}\right\| \leq \frac{1}{10}, \quad\|h(x)-h(y)\| \leq \frac{1}{100}\|x-y\|, \\
\left\|\int_{0}^{t}(k(t, s, x)-k(t, s, y)) d s\right\| \leq \frac{1}{10 e^{t}}\|x-y\|, \quad\left\|\int_{0}^{t} k(t, s, y) d s\right\| \leq \frac{1}{10+t}\|y(t)\|, \\
\left\|\int_{0}^{t}\left(h_{1}(t, s, x)-h_{1}(t, s, y)\right) d s\right\| \leq \frac{1}{10 e^{t}}\|x-y\|, \quad\left\|\int_{0}^{t} h_{1}(t, s, y) d s\right\| \leq \frac{1}{10+t}\|y(t)\|, \\
\left\|f\left(t, x_{1} y_{1}, z_{1}\right)-f\left(t, x_{2}, y_{2}, z_{2}\right)\right\| \leq \frac{1}{10+t}\left(\left\|x_{1}-x_{2}\right\|+\left\|y_{1}-y_{2}\right\|+\left\|z_{1}-z_{2}\right\|\right), \\
\quad f(t, 0,0,0)=\frac{1}{10} .
\end{array}
$$


Hence, the conditions (H1)-(H5) hold with $G_{1}=G_{2}=0.1, \quad b_{1}=b_{2}=0.01, M_{1}^{*}=0.12$, $w=0.1, C_{o}=6, N_{1}=0.1, M^{*}=0.12$, and $C_{1}=6$, thus

$$
b_{1}(1+T)+b_{2}(T-1)+\frac{w M^{*} C_{1}(1+T)}{\Gamma(\alpha+1) T^{2-\alpha}}<1 \Longleftrightarrow 0.01(2)+\frac{(0.1)(0.12) 6(2)}{\Gamma(2.5)}<1 .
$$

We conclude from the above example that the integrodifferential equation has unique solution.

\section{References}

[1] M. Amairi, M. Aoun, S. Najar, and M. N. Abdelkrim, “A constant enclosure method for validating existence and uniqueness of the solution of an initial value problem for a fractional differential equation," Applied Mathematics and Computation, vol. 217, no. 5, pp. 2162-2168, 2010.

[2] Z. Drici, F. A. McRae, and J. V. Devi, "Fractional differential equations involving causal operators," Communications in Applied Analysis., vol. 14, no. 1, pp. 81-88, 2010.

[3] S. B. Hadid, "Local and global existence theorems on differential equations of non-integer order," Journal of Fractional Calculus, vol. 7, pp. 101-105, 1995.

[4] R. W. Ibrahim, "Existence results for fractional boundary value problem," International Journal of Contemporary Mathematical Sciences, vol. 3, no. 33-36, pp. 1767-1774, 2008.

[5] S. M. Momani, "Local and global existence theorems on fractional integro-differential equations," Journal of Fractional Calculus, vol. 18, pp. 81-86, 2000.

[6] S. M. Momani and S. B. Hadid, "On the inequalities of integro-differential fractional equations," International Journal of Applied Mathematics, vol. 12, no. 1, pp. 29-37, 2003.

[7] B. Ahmad, A. Alsaedi, and B. S. Alghamdi, "Analytic approximation of solutions of the forced Duffing equation with integral boundary conditions," Nonlinear Analysis Real world Applications, vol. 9, no. 4, pp. 1727-1740, 2008.

[8] H L. Tidke, "Existence of global solutions to nonlinear mixed Volterra-Fredholm integrodifferential equations with nonlocal conditions," Electronic Journal of Differential Equations, vol. 2009, pp. No. 55-7, 2009.

[9] B. Ahmad and J. J. Nieto, "Existence results for nonlinear boundary value problems of fractional integrodifferential equations with integral boundary conditions," Boundary Value Problems, vol. 2009, Article ID 708576, 11 pages, 2009.

[10] G. M. N'Guérékata, “A Cauchy problem for some fractional abstract differential equation with non local conditions," Nonlinear Analysis: Theory , Method and Applications, vol. 70, no. 5, pp. 1873-1876, 2009.

[11] A. Anguraj, P. Karthikeyan, and J. J. Trujillo, "Existence of solutions to fractional mixed integrodifferential equations with nonlocal initial condition," Advances in Difference Equations, vol. 2011, Article ID 690653, 12 pages, 2011.

[12] A. A. Kilbas, H. M. Srivastava, and J. J. Trujillo, Theory and Applications of Fractional Differential Equations, vol. 204 of North-Holland Mathematics Studies, Elsevier Science, Amsterdam, The Netherlands, 2006.

[13] M. A. Krasnosel'skiř, "Two remarks on the method of successive approximations," Uspekhi Matematicheskikh Nauk, vol. 10, no. 1(63), pp. 123-127, 1955.

[14] I. Podlubny, Fractional Differential Equations, vol. 198 of Mathematics in Science and Engineering, Academic Press, San Diego, Calif, USA, 1999.

[15] S. G. Samko, A. A. Kilbas, and O. I. Marichev, Fractional Integrals and Derivatives, Gordon and Breach Science, Yverdon, Switzerland, 1993. 


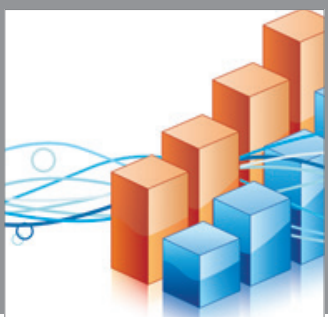

Advances in

Operations Research

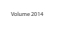

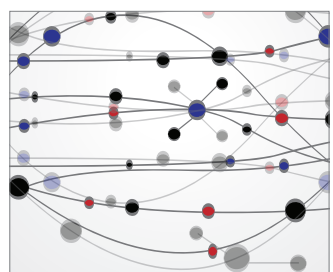

\section{The Scientific} World Journal
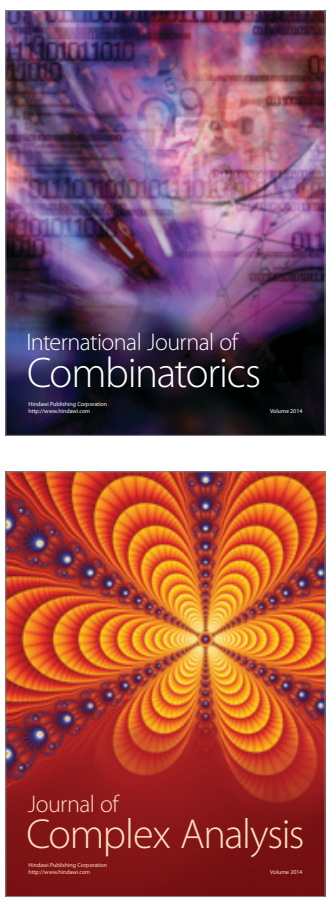

International Journal of

Mathematics and

Mathematical

Sciences
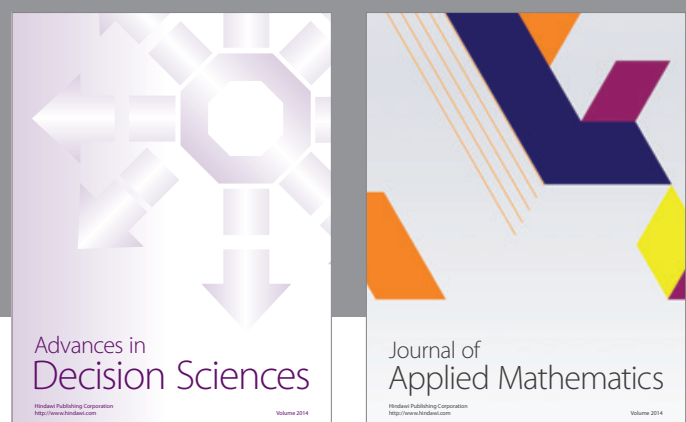

Journal of

Applied Mathematics
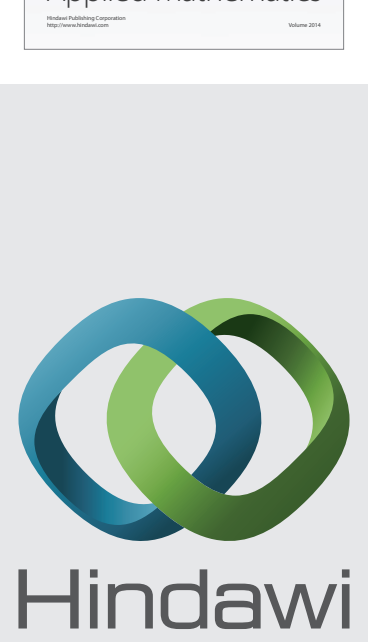

Submit your manuscripts at http://www.hindawi.com
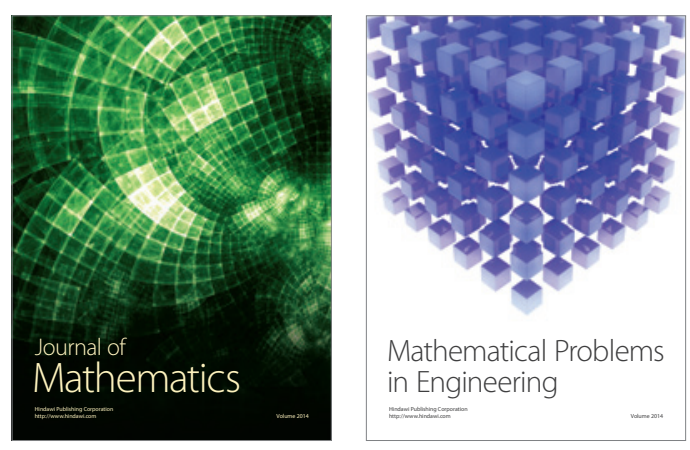

Mathematical Problems in Engineering
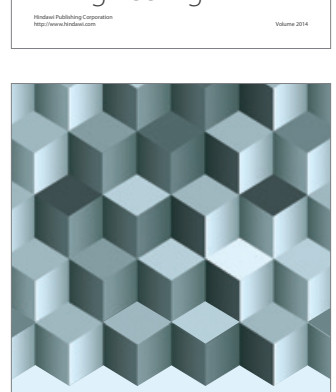

Journal of

Function Spaces
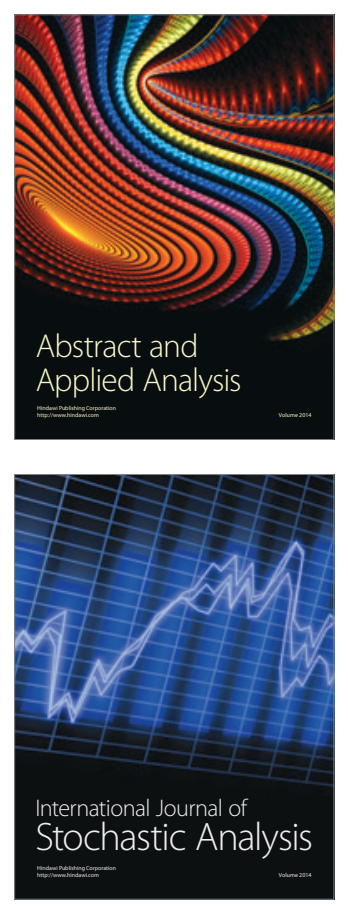

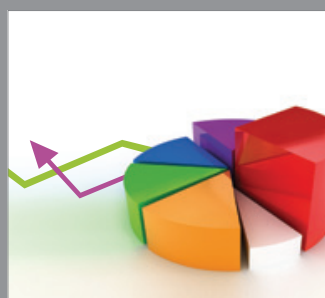

ournal of

Probability and Statistics

Promensencen
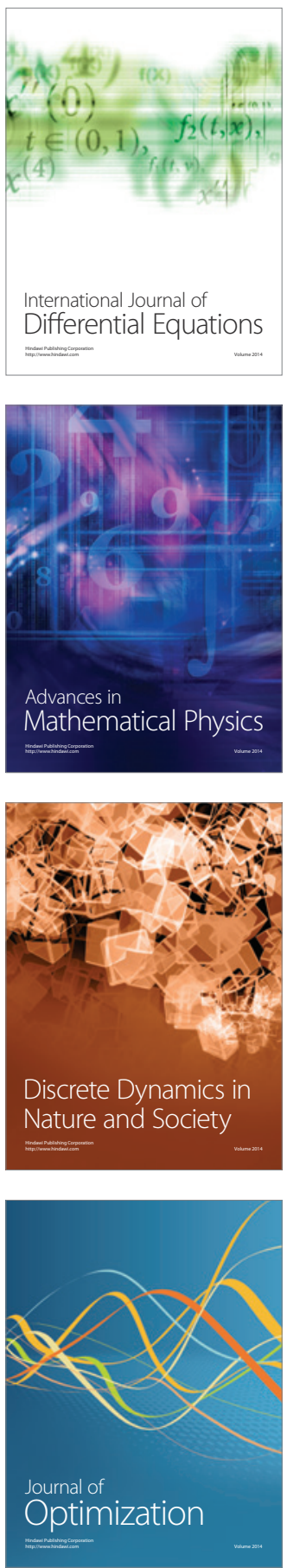